Thi $r d$ - or der perturbat i ve sol ut i ons i $n$ the Lagr angi an perturbati on theory wi th pressure II: Effect of the transverse modes

\begin{tabular}{|l|l|}
\hline 著者 & TATEKAMA TAkayuki \\
\hline $\begin{array}{l}\text { j our nal or } \\
\text { publ i cat i on t i t l e }\end{array}$ & Physi cal revi ew. [ Ser i es III . ] D \\
\hline vol une & 72 \\
\hline page range & 24005 \\
\hline year & $2005-07$ \\
\hline URL & ht t p: //hdl . handl e. net /10098/6420 \\
\hline
\end{tabular}




\title{
Third-order perturbative solutions in Lagrangian perturbation theory with pressure. II. Effect of the transverse modes
}

\author{
Takayuki Tatekawa* \\ Department of Physics, Waseda University, 3-4-1 Okubo, Shinjuku, Tokyo 169-8555, Japan \\ Advanced Research Institute for Science and Engineering, Waseda University, 3-4-1 Okubo, Shinjuku, Tokyo 169-8555, Japan \\ Department of Physics, Ochanomizu University, 2-1-1 Otsuka, Bunkyo, Tokyo 112-8610, Japan
}

(Received 18 April 2005; published 7 July 2005)

\begin{abstract}
Lagrangian perturbation theory for cosmological fluid describes structure formation in the quasinonlinear stage well. In a previous paper, we presented a third-order perturbative equation for Lagrangian perturbation with pressure. There we considered only the longitudinal modes for the first-order perturbation. In this paper, we generalize the perturbation, i.e., we consider both the longitudinal and the transverse modes for the first-order perturbation. Then we derive third-order perturbative equations and solutions.
\end{abstract}

DOI: 10.1103/PhysRevD.72.024005

PACS numbers: 04.25.Nx, 95.30.Lz, 98.65.Dx

\section{INTRODUCTION}

The structure formation scenario based on gravitational instability has been studied for a long time. The Lagrangian perturbative method for the cosmological fluid describes the nonlinear evolution of density fluctuation rather well. Zel'dovich [1] proposed a linear Lagrangian approximation for dust fluid. This approximation is called the Zel'dovich approximation (ZA) [1-9]. ZA describes the evolution of density fluctuation better than the Eulerian approximation [10-12]. Once ZA was established, the second- and the third-order perturbative solutions for dust fluid were derived [13-20].

Recently the effect of the pressure in the cosmological fluid has been considered. Buchert and Domínguez [21] showed that when the velocity dispersion is regarded as small and isotropic it produces effective "pressure" or viscosity terms. Furthermore, they posited the relation between mass density $\rho$ and pressure $P$, i.e., an "equation of state." Recently, Buchert and Domínguez [22] discussed the origin of "adhesion" in the structure formation scenario. They considered two cases, velocity dispersion and coarse graining. They named these models the Euler-JeansNewton (EJN) model and the small-size expansion (SSE) model, respectively.

In this paper, we treat only the EJN model. Following our previous paper [23], we call this model the "pressure model." Adler and Buchert [24] have formulated the Lagrangian perturbation theory for a barotropic fluid. Morita and Tatekawa [25] and Tatekawa et al. [26] solved the Lagrangian perturbation equations for a polytropic fluid up to the second order. In the aforementioned paper [23], we solved third-order perturbation equations in the simple case.

In this paper, we notice the effect of the primordial vorticity. In dust models, Buchert [16] investigated the behavior of the vorticity with the first-order Lagrangian

*Electronic address: tatekawa@gravity.phys.waseda.ac.jp perturbative equations. He showed that the primordial vorticity is amplified in proportion to the enhancement of the density fluctuations by deriving the relation between the vorticity and the density fluctuations. Barrow and Saich [13] showed the effect of the vorticity in gravitational collapse in an expanding Universe. Sasaki and Kasai [20] derived the solutions for the perturbations of both the longitudinal and the transverse modes up to third order in the EdS Universe model. Then they showed how the vorticity affects the evolution of the density fluctuation.

In the pressure model, although we showed the perturbative equations for a polytropic fluid up to the second order [25], we ignored the effect of the first-order perturbation in the transverse mode. As shown in the past paper [20], the effect of the vorticity becomes important in nonlinear evolution of the density fluctuation in the dust model. In the pressure model, the effect of the vorticity would becomes important. Because the evolution of the first-order perturbation in the transverse mode is independent of the scale of the fluctuation, the effect especially appears in small scale where the longitudinal mode shows decaying oscillation. Although we presented the thirdorder perturbative equation for the pressure model [23], we ignored the effect of the primordial vorticity. The transverse modes do not have a growing solution in the first order, we could neglect the transverse modes for the linear approximation. However, the combination of longitudinal and transverse modes do grow in third-order perturbation. Therefore when we observe the transverse mode in the initial condition, we need to estimate the effect of the transverse mode. Then we derive the perturbative solution for the simplest case, i.e., where the background is given by the Einstein-de Sitter (EdS) Universe model and the polytropic index $\gamma=4 / 3$.

This paper is organized as follows. In Sec. II, we present a Lagrangian description of basic equations for cosmological fluids. In Sec. III, we show perturbative equations and derive perturbative solutions for the pressure model up to the third order. In Sec. III A, we show the perturbative 
equations and solutions for generic polytropic fluid in the EdS Universe model. In Secs. III B and III Cwe show the perturbative equations for second- and third-order perturbation, respectively. In Sec. IV, we derive perturbative solutions. In general, it is extremely hard to solve higherorder perturbative equations. Therefore we show generic solutions for first-order perturbation only. In Secs. IVA and IV Bwe derive the perturbative solutions for second- and third-order perturbation, respectively. Here we derive the solutions only for the simplest case (EdS Universe model, $\gamma=4 / 3$ ). In Sec. V, we summarize our conclusions.

\section{BASIC EQUATIONS}

We briefly introduce the Lagrangian description for cosmological fluid. The basic equations for cosmological fluid were showed in the previous paper [23]. Here we show only the evolution equation for Lagrangian perturbation.

In Newtonian cosmology, to introduce cosmic expansion, we adopt the coordinate transformation from physical coordinates to comoving coordinates.

$$
\boldsymbol{x}=\frac{\boldsymbol{r}}{a(t)}, \quad a(t) \text { :scale factor, }
$$

where $\boldsymbol{r}$ and $\boldsymbol{x}$ are physical coordinates and comoving coordinates, respectively. The scale factor satisfies the Friedmann equations.

In the Lagrangian perturbation theory, instead of density fluctuation the displacement from homogeneous distribution is regarded as a perturbative quantity.

$$
\boldsymbol{r}=a \boldsymbol{x}=a(\boldsymbol{q}+\boldsymbol{s}(\boldsymbol{q}, t)),
$$

where $\boldsymbol{x}$ and $\boldsymbol{q}$ are the comoving Eulerian coordinates and the Lagrangian coordinates, respectively. $\boldsymbol{s}$ is the displacement vector. In the Lagrangian model, the displacement vector is regarded as a perturbative quantity. From Eq. (2), we can solve the continuous equation exactly. Then the density fluctuation is given in the formally exact form.

$$
\delta=1-J^{-1}, \quad J \equiv \operatorname{det}\left(\frac{\partial x_{i}}{\partial q_{j}}\right) .
$$

$J$ means the Jacobian of the coordinate transformation from Eulerian $\boldsymbol{x}$ to Lagrangian $\boldsymbol{q}$. Therefore, when we derive the solutions for $s$, we can know the evolution of the density fluctuation.

The peculiar velocity is given by

$$
\boldsymbol{v}=a \dot{\boldsymbol{s}} .
$$

Then we introduce the Lagrangian time derivative:

$$
\frac{d}{d t} \equiv \frac{\partial}{\partial t}+\frac{1}{a} \boldsymbol{v} \cdot \nabla_{x}
$$

Taking divergence and curl of Euler's equation, we obtain the evolution equations for the Lagrangian displacement:

$$
\begin{gathered}
\nabla_{x} \cdot\left(\ddot{\boldsymbol{s}}+2 \frac{\dot{a}}{a} \dot{\boldsymbol{s}}-\frac{\kappa \gamma \rho_{b}^{\gamma-1}}{a^{2}} J^{-\gamma} \nabla_{x} J\right) \\
=-4 \pi G \rho_{b}\left(J^{-1}-1\right), \\
\nabla_{x} \times\left(\ddot{\boldsymbol{s}}+2 \frac{\dot{a}}{a} \dot{\boldsymbol{s}}\right)=0,
\end{gathered}
$$

where (.) means the Lagrangian time derivative [Eq. (5)]. To solve the perturbative equations, we decompose the Lagrangian perturbation to the longitudinal and transverse modes:

$$
\begin{gathered}
s=\nabla S+s^{T}, \\
\nabla \cdot s^{T}=0,
\end{gathered}
$$

where $\nabla$ means the Lagrangian spacial derivative.

Here we expand the Jacobian:

$$
\begin{aligned}
J= & 1+s_{i, i}+\frac{1}{2}\left(s_{i, i} s_{j, j}-s_{i, j} s_{j, i}\right)+\operatorname{det}\left(s_{i, j}\right) \\
= & 1+\nabla^{2} S+\frac{1}{2}\left\{\left(\nabla^{2} S\right)^{2}-S_{, i j} S_{, j i}-s_{i, j}^{T} s_{j, i}^{T}\right. \\
& \left.-2 S_{, i j} s_{j, i}^{T}\right\}+\operatorname{det}\left(S_{, i j}+s_{i, j}^{T}\right) .
\end{aligned}
$$

Because Eqs. (6) and (7) include the Eulerian spacial derivative, we change to the Lagrangian spacial derivative.

$$
\begin{aligned}
\frac{\partial}{\partial x_{i}} & =\frac{\partial}{\partial q_{i}}-s_{j, i} \frac{\partial}{\partial x_{j}}=\frac{\partial}{\partial q_{i}}-s_{j, i} \frac{\partial}{\partial q_{j}}+s_{j, i} s_{k, j} \frac{\partial}{\partial x_{k}} \\
& =\frac{\partial}{\partial q_{i}}-s_{j, i} \frac{\partial}{\partial q_{j}}+s_{j, i} s_{k, j} \frac{\partial}{\partial q_{k}}+\cdots .
\end{aligned}
$$

\section{THE LAGRANGIAN PERTURBATIVE EQUATIONS}

\section{A. The first-order perturbations}

From Eqs. (6) and (7) we obtain the first-order perturbative equations:

$$
\nabla^{2}\left(\ddot{S}^{(1)}+2 \frac{\dot{a}}{a} \dot{S}^{(1)}-4 \pi G \rho_{b} S^{(1)}-\frac{\kappa \gamma \rho_{b}^{\gamma-1}}{a^{2}} \nabla^{2} S^{(1)}\right)=0,
$$

$$
\nabla\left(\ddot{\boldsymbol{s}}^{T(1)}+2 \frac{\dot{a}}{a} \dot{\boldsymbol{s}}^{T(1)}\right)=0 .
$$

The first-order solutions for the longitudinal mode depend on spacial scale. Therefore the solutions are described with a Lagrangian wave number. In this paper, we discuss only perturbative solutions in the EdS Universe model [25-27].

$$
\hat{S}^{(1)}(\boldsymbol{K}, t)=C^{+}(\boldsymbol{K}) D^{+}(\boldsymbol{K}, t)+C^{-}(\boldsymbol{K}) D^{-}(\boldsymbol{K}, t),
$$




$$
\begin{gathered}
D^{ \pm}(\boldsymbol{K}, t)= \begin{cases}t^{-1 / 6} J_{ \pm \nu}\left(A|\boldsymbol{K}| t^{-\gamma+4 / 3}\right) & \text { for } \gamma \neq \frac{4}{3}, \\
t^{-1 / 6 \pm \sqrt{25 / 36-B|\boldsymbol{K}|^{2}}} & \text { for } \gamma=\frac{4}{3},\end{cases} \\
A \equiv \frac{3 \sqrt{\kappa} \gamma(6 \pi G)^{(1-\gamma) / 2}}{|4-3 \gamma|}, \quad B \equiv \frac{4}{3} \kappa(6 \pi G)^{-1 / 3},
\end{gathered}
$$

where $J$ means Bessel function. The coefficient $\nu$ is given by $\nu=5 /(8-6 \gamma) . C^{ \pm}(\boldsymbol{K})$ is given by the initial condition.

For the transverse mode, the solutions are the same as for dust model:

$$
\mathbf{s}^{T(1)}=C^{T+}+C^{T-} t^{-1 / 3} .
$$

The transverse mode does not have a growing solution.

Therefore, when we consider only large-scale fluctuation, the longitudinal mode dominates during evolution.

According to a comparison of the first-order perturbation with a numerical simulation [28], the first-order perturbation seems rather good until the quasinonlinear regime $(\delta \sim 1)$. After that, we need to consider higherorder perturbation.

\section{B. The second-order perturbative equations}

From Eqs. (6) and (7), we obtain the second-order perturbative equations [25,27]. For the longitudinal mode, the equation becomes

$$
\nabla^{2}\left(\ddot{S}^{(2)}+2 \frac{\dot{a}}{a} \dot{S}^{(2)}-4 \pi G \rho_{b} S^{(2)}-\frac{\kappa \gamma \rho_{b}^{\gamma-1}}{a^{2}} \nabla^{2} S^{(2)}\right)=Q^{L(2)},
$$

$$
\begin{aligned}
Q^{L(2)}= & 2 \pi G \rho_{b}\left[S_{, i j}^{(1)} S_{, i j}^{(1)}-\left(\nabla^{2} S^{(1)}\right)^{2}\right]-\frac{\kappa \gamma \rho_{b}^{\gamma-1}}{a^{2}}\left\{\gamma \nabla^{2} S_{, i}^{(1)} \nabla^{2} S_{, i}^{(1)}+(\gamma-1) \nabla^{2} \nabla^{2} S^{(1)} \nabla^{2} S^{(1)}+S_{, i j k}^{(1)} S_{, i j k}^{(1)}+2 S_{, i j}^{(1)} \nabla^{2} S_{, i j}^{(1)}\right\} \\
& -\frac{\kappa \gamma \rho_{b}^{\gamma-1}}{a^{2}}\left\{S_{, i j}^{(1)} \nabla^{2} s_{i, j}^{T(1)}+2 S_{, i j k}^{(1)} s_{i, j k}^{T(1)}+\nabla^{2} S_{, i}^{(1)} \nabla^{2} s_{i}^{T(1)}+2 \nabla^{2} S_{, i j}^{(1)} s_{i, j}^{T(1)}\right\}-2 \pi G \rho_{b} s_{i, j}^{T(1)} s_{j, i}^{T(1)} \\
& -\frac{\kappa \gamma \rho_{b}^{\gamma-1}}{a^{2}}\left\{s_{i, j}^{T(1)} \nabla^{2} s_{j, i}^{T(1)}+s_{i, j k}^{T(1)} s_{j, i k}^{T(1)}\right\} .
\end{aligned}
$$

For the transverse mode, after some arrangement, we can describe as follows:

$$
\begin{gathered}
\nabla^{2}\left(\ddot{s}_{i}^{T(2)}+2 \frac{\dot{a}}{a} \dot{s}_{i}^{T(2)}\right)=Q_{i}^{T(2)} \\
Q_{i}^{T(2)}=-4 \pi G \rho_{b}\left\{s_{j, i k}^{T(1)} S_{, j k}^{(1)}+s_{j, i}^{T(1)} \nabla^{2} S_{, j}^{(1)}-\nabla^{2} s_{j}^{T(1)} S_{, i j}^{(1)}\right. \\
\left.-s_{j, k}^{T(1)} S_{, i j k}^{(1)}\right\}+\frac{\kappa \gamma \rho_{b}^{\gamma-1}}{a^{2}}\left\{S_{, i j k}^{(1)} \nabla^{2} S_{, j k}^{(1)}+S_{, i j}^{(1)} \nabla^{2} \nabla^{2} S_{, j}^{(1)}\right. \\
\left.-\nabla^{2} S_{, j}^{(1)} \nabla^{2} S_{, i j}^{(1)}-S_{, j k}^{(1)} \nabla^{2} S_{, i j k}^{(1)}\right\}+\frac{\kappa \gamma \rho_{b}^{\gamma-1}}{a^{2}} \\
\times\left(s_{j, i k}^{T(1)} \nabla^{2} S_{, j k}^{(1)}+s_{j, i}^{T(1)} \nabla^{2} \nabla^{2} S_{, j}^{(1)}-\nabla^{2} s_{j}^{T(1)} \nabla^{2} S_{, i j}^{(1)}\right. \\
\left.-s_{j, k}^{T(1)} \nabla^{2} S_{, i j k}^{(1)}\right) .
\end{gathered}
$$

Here we can note second-order transverse mode solutions. In the pressure model, even if we consider only the longitudinal mode for the first order, the second-order perturbation for the transverse mode appears. In dust model, it does not appear. Therefore, when we derive the third-order perturbative solutions, we must consider the second-order transverse mode.

\section{The third-order perturbative equations}

In the previous paper, we show the third-order perturbative equation in which only the longitudinal modes for the first-order perturbation considered [23]. The third-order perturbative equation becomes very complicated. Here we introduce scalar quantities generated by the Lagrangian perturbations.

$$
\begin{gathered}
\mu_{1}(\Psi) \equiv \nabla^{2} \Psi, \\
\mu_{2}(\boldsymbol{A}, \boldsymbol{B}) \equiv \frac{1}{2}\left((\nabla \cdot \boldsymbol{A})(\nabla \cdot \boldsymbol{B})-A_{i, j} B_{j, i}\right), \\
\mu_{2}(\boldsymbol{A}) \equiv \mu_{2}(\boldsymbol{A}, \boldsymbol{A}), \\
\mu_{3}(\boldsymbol{A}) \equiv \operatorname{det}\left(A_{i, j}\right),
\end{gathered}
$$

where $\boldsymbol{A}$ and $\boldsymbol{B}$ are vector quantities. $\Psi$ is a scalar quantity. Using these quantities, the Jacobian [Eq. (10)] is written as

$$
J=\mu_{1}(S)+\mu_{2}\left(S_{, i}+s_{i}^{T}\right)+\mu_{3}\left(S_{, i}+s_{i}^{T}\right) .
$$

First, we show the longitudinal mode equation. As in the second-order perturbative equation, we separate the terms of the third-order perturbation from the others. Then the terms consisting of the first- and the second-order perturbations are collected to the source term $Q^{L(3)}$.

$$
\nabla^{2}\left(\ddot{S}^{(3)}+2 \frac{\dot{a}}{a} \dot{S}^{(3)}-4 \pi G \rho_{b} S^{(3)}-\frac{\kappa \gamma \rho_{b}^{\gamma-1}}{a^{2}} \nabla^{2} S^{(3)}\right)=Q^{L(3)} .
$$

We consider the source term. Using Eqs. (20)-(23), the terms are written as follows: 


$$
\begin{aligned}
Q^{L(3)}= & 4 \pi G \rho_{b}\left[\mu_{1}\left(S^{(1)}\right)^{3}+\mu_{2}\left(S_{, i}^{(1)}+s_{i}^{T(1)}, S_{, i}^{(2)}+s_{i}^{T(2)}\right)+\mu_{3}\left(S_{, i}^{(1)}+s_{i}^{T(1)}\right)-\left(S_{, i j}^{(1)}+s_{j, i}^{T(1)}\right)\left(S_{, j k}^{(1)}+s_{k, j}^{T(1)}\right) S_{, i k}^{(1)}+\left(S_{, i j}^{(2)}\right.\right. \\
& \left.\left.+s_{j, i}^{T(2)}\right) S_{, i j}^{(1)}\right]+\left(S_{, i j}^{(1)}+s_{j, i}^{T(1)}\right)\left(\ddot{S}_{, i j}^{(2)}+2 \frac{\dot{a}}{a} \dot{S}_{, i j}^{(2)}+\ddot{s}_{i, j}^{T(2)}+2 \frac{\dot{a}}{a} \dot{s}_{i, j}^{T(2)}\right)+\frac{\kappa \gamma \rho_{b}^{\gamma-1}}{a^{2}}\left[\mu_{2}\left(S_{, i}^{(1)}+s_{i}^{T(1)}, S_{, i}^{(2)}+s_{i}^{T(2)}\right)+\mu_{3}\left(S_{, i}^{(1)}\right)\right. \\
& -\left(S_{, j k}^{(1)}+s_{k, j}^{T(1)}\right)\left\{\mu_{1}\left(S^{(2)}\right)+\mu_{2}\left(S_{, i}^{(1)}+s_{i}^{T(1)}\right)\right\}_{, j k}-\left\{\left(S_{, j k}^{(1)}+s_{k, j}^{T(1)}\right)\left(\mu_{1}\left(S^{(2)}\right)+\mu_{2}\left(S_{, i}^{(1)}+s_{i}^{T(1)}\right)\right)_{, k}\right\}_{, j} \\
& -\left\{\gamma \mu_{1}\left(S^{(1)}\right)\left(\mu_{1}\left(S^{(2)}\right)+\mu_{2}\left(S_{, i}^{(1)}+s_{i}^{T(1)}\right)\right)_{, j}\right\}_{, j}+\left\{\left(S_{, j k}^{(1)}+s_{k, j}^{T(1)}\right)\left(S_{, k l}^{(1)}+s_{l, k}^{T(1)}\right) \nabla^{2} S_{, l}^{(1)}-\left(S_{, j k}^{(2)}+s_{k, j}^{T(2)}\right) \nabla^{2} S_{, k}^{(1)}\right\}_{, j} \\
& -\left\{\gamma\left(\mu_{1}\left(S^{(2)}\right)+\mu_{2}\left(S_{, i}^{(1)}+s_{i}^{T(1)}\right)\right) \nabla^{2} S_{, j}^{(1)}-\frac{\gamma(\gamma+1)}{2} \mu_{1}\left(S^{(1)}\right)^{2} \mu_{1}\left(S^{(1)}\right)_{, j}\right\}_{, j}+\gamma\left\{\mu_{1}\left(S^{(1)}\right)\left(S_{, j k}^{(1)}+s_{k, j}^{T(1)}\right) \mu_{1}\left(S^{(1)}\right)_{, k}\right\}_{, j} \\
& \left.+\left(S_{, j k}^{(1)}+s_{k, j}^{T(1)}\right)\left\{\left(S_{, j l}^{(1)}+s_{l, j}^{T(1)}\right) \mu_{1}\left(S^{(1)}\right)_{, l}\right\}_{, k}+\gamma\left(S_{, j k}^{(1)}+s_{k, j}^{T(1)}\right)\left\{\mu_{1}\left(S^{(1)}\right) \mu_{1}\left(S^{(1)}\right)_{, j}\right\}_{, k}\right] .
\end{aligned}
$$

The transverse mode also seems complicated. However, if we neglect the first-order transverse mode, the evolution equation is described as

$$
\begin{aligned}
& -\nabla^{2}\left(\ddot{s}_{i}^{T(3)}+2 \frac{\dot{a}}{a} \ddot{s}_{i}^{T(3)}\right)=Q_{i}^{T(3)} \\
& Q_{i}^{T(3)}=\left\{\left(S_{, i j}^{(1)}+s_{j, i}^{T(1)}\right)\left(\ddot{S}_{, j k}^{(2)}+2 \frac{\dot{a}}{a} \dot{S}_{, j k}^{(2)}+\ddot{s}_{k, j}^{T(2)}+2 \frac{\dot{a}}{a} \dot{s}_{k, j}^{T(2)}\right)\right\}_{, k}-\left\{\left(S_{, j k}^{(1)}+s_{j, k}^{T(1)}\right)\left(\ddot{S}_{, i j}^{(2)}+2 \frac{\dot{a}}{a} \dot{S}_{, i j}^{(2)}+\ddot{s}_{i, j}^{T(2)}+2 \frac{\dot{a}}{a} \dot{s}_{i, j}^{T(2)}\right)\right\}_{, k} \\
& +\left\{\left(S_{, i j}^{(2)}+s_{i, j}^{T(2)}\right)\left(4 \pi G \rho_{b} S_{, j k}^{(1)}+\frac{\kappa \gamma \rho_{b}^{\gamma-1}}{a^{2}} \nabla^{2} S_{, j k}^{(1)}\right)\right\}_{, k}-\left\{\left(S_{, j k}^{(2)}+s_{j, k}^{T(2)}\right)\left(4 \pi G \rho_{b} S_{, i j}^{(1)}+\frac{\kappa \gamma \rho_{b}^{\gamma-1}}{a^{2}} \nabla^{2} S_{, i j}^{(1)}\right)\right\}_{, k} \\
& +\frac{\kappa \gamma \rho_{b}^{\gamma-1}}{a^{2}}\left(\nabla^{2} S_{, i l}^{(1)} S_{, j k}^{(1)} S_{, j k l}^{(1)}+\nabla^{2} S_{, i k}^{(1)} \nabla^{2} S_{, j}^{(1)} S_{, j k}^{(1)}+\nabla^{2} S_{, i k l}^{(1)} S_{, j k}^{(1)} S_{, j l}^{(1)}-S_{, i j k}^{(1)} S_{, j l}^{(1)} \nabla^{2} S_{, k l}^{(1)}-S_{, i j}^{(1)} S_{, j k l}^{(1)} \nabla^{2} S_{, k l}^{(1)}\right. \\
& \left.-S_{, i j}^{(1)} S_{, j k}^{(1)} \nabla^{2} S_{, k}^{(1)}\right)+4 \pi G \rho_{b}\left[\nabla^{2} s_{k}^{T(1)} S_{, i j}^{(1)} S_{, j k}^{(1)}-s_{l, i j}^{T(1)} S_{, k l}^{(1)} S_{, j k}^{(1)}+s_{k, j}^{T(1)}\left(\nabla^{2} S_{, j}^{(1)} S_{, i k}^{(1)}-\nabla^{2} S_{, k}^{(1)} S_{, i j}^{(1)}+S_{, j l}^{(1)} S_{, i k l}^{(1)}\right.\right. \\
& \left.\left.-S_{, i j l}^{(1)} S_{, k l}^{(1)}+S_{, i l}^{(1)} S_{, j k l}^{(1)}+S_{, k l}^{(1)} S_{, i j l}^{(1)}\right)-s_{j, i}^{T(1)}\left(S_{, j k l}^{(1)} S_{, j k}^{(1)}+S_{, j k}^{(1)} \nabla^{2} S_{, k}^{(1)}\right)+s_{k, j l}^{T(1)}\left(S_{, j l}^{(1)} S_{, i k}^{(1)}-S_{, i l}^{(1)} S_{, j k}^{(1)}\right)\right] \\
& +\frac{\kappa \gamma \rho_{b}^{\gamma-1}}{a^{2}}\left[\nabla^{2} s_{k}^{T(1)} S_{, j k}^{(1)} \nabla^{2} S_{, i j}^{(1)}-s_{l, i j}^{T(1)} S_{, k l}^{(1)} \nabla^{2} S_{, j k}^{(1)}+s_{k, j}^{T(1)}\left(\nabla^{2} S_{, j}^{(1)} \nabla^{2} S_{, i k}^{(1)}-\nabla^{2} \nabla^{2} S_{, k}^{(1)} S_{, i j}^{(1)}+S_{, j l}^{(1)} \nabla^{2} S_{, i k l}^{(1)}\right.\right. \\
& \left.\left.-S_{, i j l}^{(1)} \nabla^{2} S_{, k l}^{(1)}+S_{, j k l}^{(1)} \nabla^{2} S_{, i l}^{(1)}+S_{, k l}^{(1)} \nabla^{2} S_{, i j l}^{(1)}\right)-s_{j, i}^{T(1)}\left(S_{, j k l}^{(1)} \nabla^{2} S_{, k l}^{(1)}+S_{, j k}^{(1)} \nabla^{2} \nabla^{2} S_{, k}^{(1)}\right)+s_{k, j l}^{T(1)}\left(S_{, j l}^{(1)} \nabla^{2} S_{, i k}^{(1)}-S_{, i l}^{(1)} \nabla^{2} S_{, j k}^{(1)}\right)\right] \\
& +4 \pi G \rho_{b}\left[-\left(s_{l, i j}^{T(1)} s_{k, l}^{T(1)}+s_{l, i}^{T(1)} s_{k, j l}^{T(1)}\right) S_{, j k}^{(1)}-s_{k, i}^{T(1)} s_{j, k}^{T(1)} \nabla^{2} S_{, j}^{(1)}+\left(\nabla^{2} s_{k}^{T(1)} s_{j, k}^{T(1)}+s_{l, k}^{T(1)} s_{j, k l}^{T(1)}\right) S_{, i j}^{(1)}+s_{l, j}^{T(1)} s_{k, l}^{T(1)} S_{, i j k}^{(1)}\right] \\
& +\frac{\kappa \gamma \rho_{b}^{\gamma-1}}{a^{2}}\left[-\left(s_{l, i j}^{T(1)} s_{k, l}^{T(1)}+s_{l, i}^{T(1)} s_{k, j l}^{T(1)}\right) \nabla^{2} S_{, j k}^{(1)}-s_{k, i}^{T(1)} s_{j, k}^{T(1)} \nabla^{2} \nabla^{2} S_{, j}^{(1)}+\left(\nabla^{2} s_{k}^{T(1)} s_{j, k}^{T(1)}+s_{l, k}^{T(1)} s_{j, k l}^{T(1)}\right) \nabla^{2} S_{, i j}^{(1)}\right. \\
& \left.+s_{l, j}^{T(1)} s_{k, l}^{T(1)} \nabla^{2} S_{, i j k}^{(1)}\right] \text {. }
\end{aligned}
$$

\section{LAGRANGIAN PERTURBATIVE SOLUTIONS FOR HIGHER-ORDER PERTURBATION}

\section{A. The second-order perturbative solutions: The sim- plest case}

The second-order solutions are formally written as follows:

$$
\begin{gathered}
\hat{S}^{(2)}=-\frac{1}{|\boldsymbol{K}|^{2}} \int^{t} d t^{\prime} G\left(\boldsymbol{K}, t, t^{\prime}\right) \hat{Q}^{L(2)}(\boldsymbol{K}, t), \\
\hat{s}^{T(2)}=-\frac{1}{|\boldsymbol{K}|^{2}} \int^{t} d t^{\prime} G^{T}\left(t, t^{\prime}\right) \hat{Q}^{T(2)}(\boldsymbol{K}, t), \\
G^{T}\left(t, t^{\prime}\right)=3\left(t^{\prime}-t^{-1 / 3} t^{\prime 4 / 3}\right) .
\end{gathered}
$$

$G^{L}\left(\boldsymbol{K}, t, t^{\prime}\right)$ depends on the "equation of state." If $\gamma \neq 4 / 3$ and $\nu=5 /(8-6 \gamma)$ is not an integer, we have

$$
\begin{aligned}
G^{L}\left(\boldsymbol{K}, t, t^{\prime}\right)= & -\frac{\pi}{2 \sin \nu \pi}\left(-\gamma+\frac{4}{3}\right)^{-1} t^{-1 / 6} t^{\eta / 6} \\
& \times\left[J_{-\nu}\left(A|\boldsymbol{K}| t^{-\gamma+4 / 3}\right) J_{\nu}\left(A|\boldsymbol{K}| t^{\prime-\gamma+4 / 3}\right)\right. \\
& \left.-J_{\nu}\left(A|\boldsymbol{K}| t^{-\gamma+4 / 3}\right) J_{-\nu}\left(A|\boldsymbol{K}| t^{\prime-\gamma+4 / 3}\right)\right],
\end{aligned}
$$

and if $\gamma=4 / 3$,

$$
\begin{aligned}
& G^{L}\left(\boldsymbol{K}, t, t^{\prime}\right)=-\frac{1}{2 b(\boldsymbol{K})} t^{-1 / 6} t^{7 / 6}\left(t^{-b(\boldsymbol{K})} t^{\prime b(\boldsymbol{K})}\right. \\
&\left.-t^{b(\boldsymbol{K})} t^{\prime-b(\boldsymbol{K})}\right) \\
& b(\boldsymbol{K}) \equiv \sqrt{\frac{25}{36}-B|\boldsymbol{K}|^{2}}
\end{aligned}
$$


The second-order perturbative solutions for the case of $\gamma=4 / 3$ in the EdS universe model has already been derived by Morita and Tatekawa [25]. The solutions are described by

$$
\begin{gathered}
\hat{S}^{(2)}(\boldsymbol{K})=\int d \boldsymbol{K}^{\prime}\left(\Xi_{1}^{L}\left(\boldsymbol{K}, \boldsymbol{K}^{\prime}\right) E_{1}^{L}\left(\boldsymbol{K}, \boldsymbol{K}^{\prime}, t\right)\right. \\
+\Xi_{2 i}^{L}\left(\boldsymbol{K}, \boldsymbol{K}^{\prime}\right) E_{2 i}^{L}\left(\boldsymbol{K}, \boldsymbol{K}^{\prime}, t\right) \\
\left.+\Xi_{3 i j}^{L}\left(\boldsymbol{K}, \boldsymbol{K}^{\prime}\right) E_{3 i j}^{L}\left(\boldsymbol{K}, \boldsymbol{K}^{\prime}, t\right)\right) \\
\hat{s}_{i}^{T(2)}(\boldsymbol{K})=\int d \mathbf{K}^{\prime}\left(\Xi_{1 i}^{T}\left(\boldsymbol{K}, \boldsymbol{K}^{\prime}\right) E_{1}^{T}\left(\boldsymbol{K}, \boldsymbol{K}^{\prime}, t\right)\right. \\
\left.\quad+\Xi_{2 i j}^{T}\left(\boldsymbol{K}, \boldsymbol{K}^{\prime}\right) E_{2 j}^{T}\left(\boldsymbol{K}, \boldsymbol{K}^{\prime}, t\right)\right), \\
\Xi_{1}^{L}\left(\boldsymbol{K}, \boldsymbol{K}^{\prime}\right)=-\frac{1}{(2 \pi)^{3}} \frac{1}{|\boldsymbol{K}|^{2}}\left[\frac { 1 } { 3 } \left\{\left(\boldsymbol{K}^{\prime} \cdot\left(\boldsymbol{K}-\boldsymbol{K}^{\prime}\right)\right)^{2}\right.\right. \\
\left.-\left|\boldsymbol{K}^{\prime}\right|^{2}\left|\boldsymbol{K}-\boldsymbol{K}^{\prime}\right|^{2}\right\}+B\left\{\frac{4}{3}\left|\boldsymbol{K}^{\prime}\right|^{2} \mid \boldsymbol{K}\right. \\
-\left.\boldsymbol{K}^{\prime}\right|^{2}\left(\boldsymbol{K}^{\prime} \cdot\left(\boldsymbol{K}-\boldsymbol{K}^{\prime}\right)\right)+\frac{1}{3}\left|\boldsymbol{K}^{\prime}\right|^{4}\left|\boldsymbol{K}-\boldsymbol{K}^{\prime}\right|^{2} \\
+\left(\left(\boldsymbol{K}^{\prime} \cdot\left(\boldsymbol{K}-\boldsymbol{K}^{\prime}\right)\right)^{3}+2 \mid \boldsymbol{K}\right. \\
\left.\left.\left.-\left.\boldsymbol{K}^{\prime}\right|^{2}\left(\boldsymbol{K}^{\prime} \cdot\left(\boldsymbol{K}-\boldsymbol{K}^{\prime}\right)\right)^{2}\right)\right\}\right],
\end{gathered}
$$

$$
\begin{aligned}
\Xi_{3 i j}^{L}\left(\boldsymbol{K}, \boldsymbol{K}^{\prime}\right)= & -\frac{1}{(2 \pi)^{3}} \frac{1}{|\boldsymbol{K}|^{2}}\left[\frac{1}{3}\left(\boldsymbol{K}^{\prime} \cdot\left(\boldsymbol{K}-\boldsymbol{K}^{\prime}\right)\right)\right. \\
& \left.-B\left\{\left|\boldsymbol{K}-\boldsymbol{K}^{\prime}\right|^{2}+\left(\boldsymbol{K}^{\prime} \cdot\left(\boldsymbol{K}-\boldsymbol{K}^{\prime}\right)\right)\right\}\right] \\
& \times K_{i}^{\prime}\left(K_{j}-K_{j}^{\prime}\right),
\end{aligned}
$$

$$
\begin{aligned}
\Xi_{1 i}^{T}\left(\boldsymbol{K}, \boldsymbol{K}^{\prime}\right)= & -\frac{i}{(2 \pi)^{3}} \frac{B}{|\boldsymbol{K}|^{2}}\left|\boldsymbol{K}-\boldsymbol{K}^{\prime}\right|^{2}\left(\boldsymbol{K}^{\prime} \cdot\left(\boldsymbol{K}-\boldsymbol{K}^{\prime}\right)\right) \\
& \times\left[\left(\boldsymbol{K}^{\prime} \cdot\left(\boldsymbol{K}-\boldsymbol{K}^{\prime}\right)\right) K_{i}^{\prime}+\left|\boldsymbol{K}-\boldsymbol{K}^{\prime}\right|^{2} K_{i}^{\prime}\right. \\
& -\left|\boldsymbol{K}^{\prime}\right|^{2}\left(K_{i}-K_{i}^{\prime}\right)-\left(\boldsymbol{K}^{\prime} \cdot\left(\boldsymbol{K}-\boldsymbol{K}^{\prime}\right)\right) \\
& \left.\times\left(K_{i}-K_{i}^{\prime}\right)\right],
\end{aligned}
$$

$$
E_{2 j}^{T}\left(\boldsymbol{K}, \boldsymbol{K}^{\prime}, t\right) \propto \sum_{(\oplus= \pm)}\left[\frac{C^{T \oplus}\left(\boldsymbol{K}^{\prime}\right) C^{+}\left(\boldsymbol{K}-\boldsymbol{K}^{\prime}\right)}{b(\boldsymbol{K})^{2}-\frac{1}{36}} t^{-1 / 6 \oplus b(\boldsymbol{K})}+\frac{C^{T \oplus}\left(\boldsymbol{K}^{\prime}\right) C^{-}\left(\boldsymbol{K}-\boldsymbol{K}^{\prime}\right)}{\left(b\left(\boldsymbol{K}-\boldsymbol{K}^{\prime}\right) \oplus\left(-\frac{1}{2}\right)\right)\left(b\left(\boldsymbol{K}-\boldsymbol{K}^{\prime}\right) \oplus\left(-\frac{1}{6}\right)\right)} t^{-1 / 2 \oplus b\left(\boldsymbol{K}-\boldsymbol{K}^{\prime}\right)}\right] .
$$

where $\sum_{(\oplus= \pm)}$ means 


$$
\sum_{(\oplus= \pm)}\left(\alpha^{\oplus} \oplus \beta\right) \equiv\left(\alpha^{+}+\beta\right)+\left(\alpha^{-}-\beta\right) .
$$

$E_{2}^{L}$ and $E_{1}^{T}$ appears only in the pressure model. If we ignore the effect of the pressure, only $E_{1}^{L}, E_{3}^{L}$, and $E_{2}^{T}$ appear. From these results, it is apparent that the pressure does affect the evolution of the higher-order perturbation.

\section{B. The third-order perturbative solutions: The simplest case}

As in the first- and the second-order solutions, the thirdorder solutions are described with the Lagrangian wave number. Following the method used in the second-order solutions, the third-order solution is given by this integration:

$$
\begin{gathered}
\hat{S}^{(3)}=-\frac{1}{|\boldsymbol{K}|^{2}} \int_{t_{0}}^{t} d t^{\prime} G\left(\boldsymbol{K}, t, t^{\prime}\right) \hat{Q}^{L(3)}(\boldsymbol{K}, t), \\
\hat{s}^{T(3)}=-\frac{1}{|\boldsymbol{K}|^{2}} \int_{t_{0}}^{t} d t^{\prime} G^{T}\left(t, t^{\prime}\right) \hat{Q}^{T(3)}(\boldsymbol{K}, t) .
\end{gathered}
$$

In the previous paper [23], we showed the third-order perturbative solutions for the simplest case, the case of $\gamma=4 / 3$ in the EdS Universe model. In this case, the contributions of the gravitational terms and the pressure terms become identical:

$$
4 \pi G \rho_{b}, \frac{\kappa \gamma \rho_{b}^{\gamma-1}}{a^{2}} \propto a^{-3} .
$$

In addition to these conditions, we considered only the longitudinal modes in the first-order perturbation. Here we consider both the longitudinal and the transverse modes in the first-order perturbation. As we show in Sec. III C, the third-order perturbative equations become more complicated. To avoid complexity, we estimate only time evolution in the third-order perturbative solutions $F(t)$ :

$$
F(t) \propto t^{\alpha} .
$$

The third-order perturbation is affected from the first- and the second-order perturbation via source terms $Q^{L}$ and $Q^{T}$ :

$$
\begin{aligned}
Q^{L(3)}= & Q^{L(3)}\left(S^{(1)} * S^{(2)}, S^{(1)} * s^{T(2)}, s^{T(1)} * S^{(2)}, s^{T(1)}\right. \\
& * s^{T(2)}, S^{(1)} * S^{(1)} * S^{(1)}, S^{(1)} * S^{(1)} * s^{T(1)}, S^{(1)} \\
& \left.* s^{T(1)} * s^{T(1)}, s^{T(1)} * s^{T(1)} * s^{T(1)}\right), \\
Q^{T(3)}= & Q^{T(3)}\left(S^{(1)} * S^{(2)}, S^{(1)} * s^{T(2)}, s^{T(1)} * S^{(2)}, s^{T(1)}\right. \\
& * s^{T(2)}, S^{(1)} * S^{(1)} * S^{(1)}, S^{(1)} * S^{(1)} * s^{T(1)}, S^{(1)} \\
& \left.* s^{T(1)} * s^{T(1)}\right),
\end{aligned}
$$

where $*$ means convolution.

In Secs. III A and IVAwe showed the time dependence of the perturbative solutions. Here we arrange and list again:

$$
\begin{gathered}
D^{ \pm}(\boldsymbol{K}) \propto t^{-1 / 6 \pm b(\boldsymbol{K})}, \\
D^{T \pm} \propto t^{-1 / 6 \pm 1 / 6}, \\
E_{1}^{L}\left(\boldsymbol{K}, \boldsymbol{K}^{\prime}\right) \propto t^{-1 / 3 \pm b\left(\boldsymbol{K}^{\prime}\right) \pm b\left(\boldsymbol{K}-\boldsymbol{K}^{\prime}\right)}, \\
E_{2}^{L}\left(\boldsymbol{K}, \boldsymbol{K}^{\prime}\right) \propto t^{-1 / 3 \pm 1 / 6 \pm b\left(\boldsymbol{K}-\boldsymbol{K}^{\prime}\right)},
\end{gathered}
$$

\begin{tabular}{|c|c|c|c|}
\hline The convolution & The contribution & The indices $\alpha$ & \\
\hline$S^{(1)}\left(\boldsymbol{K}^{\prime \prime}\right) * S^{(2)}\left(\boldsymbol{K}-\boldsymbol{K}^{\prime \prime}\right)$ & $\begin{array}{l}D E_{1}^{L} \\
D E_{2}^{L} \\
D E_{3}^{L}\end{array}$ & $\begin{array}{l}-\frac{1}{2} \pm b_{1} \pm b_{2} \pm b_{3} \\
-\frac{1}{2} \pm \frac{1}{6} \pm b_{1} \pm b_{4} \\
-\frac{1}{2} \pm \frac{1}{6} \pm \frac{1}{6} \pm b_{1}\end{array}$ & $\mathrm{P}$ \\
\hline$S^{(1)}\left(\boldsymbol{K}^{\prime \prime}\right) * s^{T(2)}\left(\boldsymbol{K}-\boldsymbol{K}^{\prime \prime}\right)$ & $\begin{array}{l}D E_{1}^{T} \\
D E_{2}^{T}\end{array}$ & $\begin{array}{l}-\frac{1}{2} \pm b_{1} \pm b_{2} \pm b_{3} \\
-\frac{1}{2} \pm \frac{1}{6} \pm b_{1} \pm b_{4}\end{array}$ & $\mathrm{P}$ \\
\hline$s^{T(1)}\left(\boldsymbol{K}^{\prime \prime}\right) * S^{(2)}\left(\boldsymbol{K}-\boldsymbol{K}^{\prime \prime}\right)$ & $\begin{array}{l}D^{T} E_{1}^{L} \\
D^{T} E_{2}^{L} \\
D^{T} E_{3}^{L}\end{array}$ & $\begin{array}{l}-\frac{1}{2} \pm \frac{1}{6} \pm b_{2} \pm b_{3} \\
-\frac{1}{2} \pm \frac{1}{6} \pm \frac{1}{6} \pm b_{4} \\
-\frac{1}{2} \pm \frac{1}{6} \pm \frac{1}{6} \pm \frac{1}{6}\end{array}$ & $\mathrm{P}$ \\
\hline$s^{T(1)}\left(\boldsymbol{K}^{\prime \prime}\right) * s^{T(2)}\left(\boldsymbol{K}-\boldsymbol{K}^{\prime \prime}\right)$ & $\begin{array}{l}D^{T} E_{1}^{T} \\
D^{T} E_{2}^{T}\end{array}$ & $\begin{array}{l}-\frac{1}{2} \pm \frac{1}{6} \pm b_{2} \pm b_{3} \\
-\frac{1}{2} \pm \frac{1}{6} \pm \frac{1}{6} \pm b_{4}\end{array}$ & $\mathrm{P}$ \\
\hline$S^{(1)}\left(\boldsymbol{K}^{\prime \prime}\right) * S^{(1)}\left(\boldsymbol{K}^{\prime}-\boldsymbol{K}^{\prime \prime}\right) * S^{(1)}\left(\boldsymbol{K}-\boldsymbol{K}^{\prime}\right)$ & $D D D$ & $-\frac{1}{2} \pm b_{1} \pm b_{2} \pm b_{3}$ & \\
\hline$s^{T(1)}\left(\boldsymbol{K}^{\prime \prime}\right) * S^{(1)}\left(\boldsymbol{K}^{\prime}-\boldsymbol{K}^{\prime \prime}\right) * S^{(1)}\left(\boldsymbol{K}-\boldsymbol{K}^{\prime}\right)$ & $D^{T} D D$ & $-\frac{1}{2} \pm \frac{1}{6} \pm b_{2} \pm b_{3}$ & \\
\hline$s^{T(1)}\left(\boldsymbol{K}^{\prime \prime}\right) * s^{T(1)}\left(\boldsymbol{K}^{\prime}-\boldsymbol{K}^{\prime \prime}\right) * S^{(1)}\left(\boldsymbol{K}-\boldsymbol{K}^{\prime}\right)$ & $D^{T} D^{T} D$ & $-\frac{1}{2} \pm \frac{1}{6} \pm \frac{1}{6} \pm b_{3}$ & $\mathrm{P}$ \\
\hline$s^{T(1)}\left(\boldsymbol{K}^{\prime \prime}\right) * s^{T(1)}\left(\boldsymbol{K}^{\prime}-\boldsymbol{K}^{\prime \prime}\right) * s^{T(1)}\left(\boldsymbol{K}-\boldsymbol{K}^{\prime}\right)$ & $D^{T} D^{T} D^{T}$ & $-\frac{1}{2} \pm \frac{1}{6} \pm \frac{1}{6} \pm \frac{1}{6}$ & \\
\hline
\end{tabular}

TABLE I. The time dependence of the third-order perturbative solutions in the longitudinal modes (the case of $\gamma=4 / 3$ in the EdS universe model). The contribution means the time component in the source terms. The indices of the time evolution $\alpha$ [Eq. (51)] depend on the convolution in the source terms $Q^{L(3)}$. Here we define $b_{1} \equiv b\left(\boldsymbol{K}^{\prime \prime}\right), b_{2} \equiv b\left(\boldsymbol{K}^{\prime}-\boldsymbol{K}^{\prime \prime}\right), b_{3} \equiv$ $b\left(\boldsymbol{K}-\boldsymbol{K}^{\prime}\right), b_{4} \equiv b\left(\boldsymbol{K}-\boldsymbol{K}^{\prime \prime}\right)$. "P" denotes that the contribution does not appear in the dust model. 
TABLE II. The time dependence of the third-order perturbative solutions in the transverse modes (the case of $\gamma=4 / 3$ in the EdS universe model). The contribution means the time component in the source terms. The indices of the time evolution $\alpha$ [Eq. (51)] depend on the convolution in the source terms $Q^{L(3)}$. Here we define $b_{1} \equiv b\left(\boldsymbol{K}^{\prime \prime}\right), b_{2} \equiv b\left(\boldsymbol{K}^{\prime}-\boldsymbol{K}^{\prime \prime}\right), b_{3} \equiv$ $b\left(\boldsymbol{K}-\boldsymbol{K}^{\prime}\right), b_{4} \equiv b\left(\boldsymbol{K}-\boldsymbol{K}^{\prime \prime}\right)$. P denotes that the contribution does not appear in the dust model.

\begin{tabular}{lcll}
\hline \hline The convolution & The contribution & The indices $\alpha$ & \\
\hline$S^{(1)}\left(\boldsymbol{K}^{\prime \prime}\right) * S^{(2)}\left(\boldsymbol{K}-\boldsymbol{K}^{\prime \prime}\right)$ & $D E_{1}^{L}$ & $-\frac{1}{2} \pm b_{1} \pm b_{2} \pm b_{3}$ & \\
& $D E_{2}^{L}$ & $-\frac{1}{2} \pm \frac{1}{6} \pm b_{1} \pm b_{4}$ & $\mathrm{P}$ \\
& $D E_{3}^{L}$ & $-\frac{1}{2} \pm \frac{1}{6} \pm \frac{1}{6} \pm b_{1}$ & \\
$S^{(1)}\left(\boldsymbol{K}^{\prime \prime}\right) * s^{T(2)}\left(\boldsymbol{K}-\boldsymbol{K}^{\prime \prime}\right)$ & $D E_{1}^{T}$ & $-\frac{1}{2} \pm b_{1} \pm b_{2} \pm b_{3}$ & $\mathrm{P}$ \\
& $D E_{2}^{T}$ & $-\frac{1}{2} \pm \frac{1}{6} \pm b_{1} \pm b_{4}$ & \\
$s^{T(1)}\left(\boldsymbol{K}^{\prime \prime}\right) * S^{(2)}\left(\boldsymbol{K}-\boldsymbol{K}^{\prime \prime}\right)$ & $D^{T} E_{1}^{L}$ & $-\frac{1}{2} \pm \frac{1}{6} \pm b_{2} \pm b_{3}$ & \\
& $D^{T} E_{2}^{L}$ & $-\frac{1}{2} \pm \frac{1}{6} \pm \frac{1}{6} \pm b_{4}$ & $\mathrm{P}$ \\
& $D^{T} E_{3}^{L}$ & $-\frac{1}{2} \pm \frac{1}{6} \pm \frac{1}{6} \pm \frac{1}{6}$ & \\
$s^{T(1)}\left(\boldsymbol{K}^{\prime \prime}\right) * s^{T(2)}\left(\boldsymbol{K}-\boldsymbol{K}^{\prime \prime}\right)$ & $D^{T} E_{1}^{T}$ & $-\frac{1}{2} \pm \frac{1}{6} \pm b_{2} \pm b_{3}$ & $\mathrm{P}$ \\
$S^{(1)}\left(\boldsymbol{K}^{\prime \prime}\right) * S^{(1)}\left(\boldsymbol{K}^{\prime}-\boldsymbol{K}^{\prime \prime}\right) * S^{(1)}\left(\boldsymbol{K}-\boldsymbol{K}^{\prime}\right)$ & $D^{T} E_{2}^{T}$ & $-\frac{1}{2} \pm \frac{1}{6} \pm \frac{1}{6} \pm b_{4}$ & \\
$s^{T(1)}\left(\boldsymbol{K}^{\prime \prime}\right) * S^{(1)}\left(\boldsymbol{K}^{\prime}-\boldsymbol{K}^{\prime \prime}\right) * S^{(1)}\left(\boldsymbol{K}-\boldsymbol{K}^{\prime}\right)$ & $D D D$ & $-\frac{1}{2} \pm b_{1} \pm b_{2} \pm b_{3}$ & $\mathrm{P}$ \\
$s^{T(1)}\left(\boldsymbol{K}^{\prime \prime}\right) * s^{T(1)}\left(\boldsymbol{K}^{\prime}-\boldsymbol{K}^{\prime \prime}\right) * S^{(1)}\left(\boldsymbol{K}-\boldsymbol{K}^{\prime}\right)$ & $D^{T} D D$ & $-\frac{1}{2} \pm \frac{1}{6} \pm b_{2} \pm b_{3}$ & $\mathrm{P}$ \\
\hline \hline
\end{tabular}

$$
\begin{gathered}
E_{3}^{L}\left(\boldsymbol{K}, \boldsymbol{K}^{\prime}\right) \propto t^{-1 / 3 \pm 1 / 6 \pm 1 / 6}, \\
E_{1}^{T}\left(\boldsymbol{K}, \boldsymbol{K}^{\prime}\right) \propto t^{-1 / 3 \pm b\left(\boldsymbol{K}^{\prime}\right) \pm b\left(\boldsymbol{K}-\boldsymbol{K}^{\prime}\right)}, \\
E_{2}^{T}\left(\boldsymbol{K}, \boldsymbol{K}^{\prime}\right) \propto t^{-1 / 3 \pm 1 / 6 \pm b\left(\boldsymbol{K}-\boldsymbol{K}^{\prime}\right)} .
\end{gathered}
$$

From Eq. (34), if we take a limit of weak pressure $(P \rightarrow 0)$, $b(\boldsymbol{K})$ converges to

$$
b(K) \rightarrow \frac{5}{6}
$$

Here we notice the relation between $E_{1}^{L}$ and $E_{1}^{T}$, i.e., time evolution in both the longitudinal and the transverse modes. Both of these terms are generated from $S^{(1)} * S^{(1)}$ in $Q^{L(2)}$ and $Q^{T(2)}$. Therefore if the convolution in the source terms in both modes are identical, the evolutions will also be identical.

Tables I and II show the time dependence of the thirdorder perturbative solutions. When the dust model [20] and the pressure model are compared, we notice that several terms in the third-order perturbative solutions do not appear in the dust model. For example, the contribution of the term $D E_{1}^{T}$, which is proportional to $D E_{1}^{L}$, i.e., one of most dominant term, appears only in the pressure model.

\section{SUMMARY AND CONCLUDING REMARKS}

In this paper, we showed the third-order Lagrangian perturbative equations for the cosmological fluid with pressure. Then we derived third-order perturbative solutions for a simple case.

In the pressure model, we must notice one other point. The longitudinal mode in the first-order perturbation depends on the scale (or the wave number) of the fluctuation.
On the other hand, the transverse mode in the first-order perturbation is independent of the scale of the fluctuation. In the dust model, because the transverse mode does not have growing modes, even if the primordial vorticity exists, we can simply ignore this mode. However in the pressure model, we cannot ignore it. The growing factor in the longitudinal mode depends on the scale of the fluctuation; when we consider small-scale fluctuation, the transverse mode dominates.

The transverse modes do not appear in a spacial onedimensional model. In two- or three-dimensional models, the computation of the perturbation becomes huge. Especially, in the third-order perturbation, enormous mode-coupling computation is required. Even if in a onedimensional model [23], the computation of the third-order approximation required a long time. When we compute the third-order approximation in the generic case, we will apply Monte Carlo integration [29] for the computation of the mode coupling.

Here we estimate the importance of the transverse mode in the pressure model. For simplicity, we consider only the EdS Universe model. In past papers [25,26], we showed that when the polytropic index $\gamma$ is less than $4 / 3$, all longitudinal modes oscillate and decay. On the other hand, when the polytropic index $\gamma$ is more than 4/3, all longitudinal modes grow as those in the dust model do. The case of $\gamma=4 / 3$ is critical. We consider the index of the time component in the first-order perturbation [Eq. (14)]. If the Lagrangian wave number $\boldsymbol{K}$ satisfies the condition

$$
\frac{25}{36}-B|\boldsymbol{K}|^{2}>\frac{1}{36},
$$

i.e., 


$$
|K|<\sqrt{\frac{2}{3 B}}=\frac{2 \sqrt{6}}{5} K_{J} \simeq 0.98 K_{J},
$$

the longitudinal modes dominate during evolution. Therefore when we consider small-scale $\left(|\boldsymbol{K}|>(2 \sqrt{6} / 5) K_{J}\right)$ fluctuation with primordial vorticity, we must consider the evolution of the transverse modes.

Recently several dark matter models have been proposed [30]. In several models, special interaction affects the avoidance of matter concentration. In general, this interaction is introduced by scattering cross section. Instead of the cross section, if the interaction in some kind of dark matter can be described by the effective pressure, we can examine the behavior of the density fluctuation in a quasinonlinear stage. Furthermore, when we compare the observations and the structure that is formed by using the pressure model, we can delimit the nature of the dark matter. In the future, when we consider the evolution of the density fluctuation or the peculiar velocity in even high-density regions, although these seem highly complicated, the third-order perturbative solutions may become useful.

\section{ACKNOWLEDGMENTS}

We are grateful to Kei-ichi Maeda for his continuous encouragement. We would like to thank Peter Musolf for checking of English writing of this paper. This work was supported by the Grant-in-Aid for Scientific Research Fund of the Ministry of Education, Culture, Sports, Science and Technology [Young Scientists (B) 16740152)].
[1] Ya. B. Zel'dovich, Astron. Astrophys. 5, 84 (1970).

[2] V.I. Arnol'd, S.F. Shandarin, and Ya. B. Zel'dovich, Geophys. Astrophys. Fluid Dyn. 20, 111 (1982).

[3] S. F. Shandarin and Ya. B. Zel'dovich, Rev. Mod. Phys. 61, 185 (1989).

[4] T. Buchert, Astron. Astrophys. 223, 9 (1989).

[5] P. Coles and F. Lucchin, Cosmology: The Origin and Evolution of Cosmic Structure (John Wiley \& Sons, Chichester, 1995).

[6] V. Sahni and P. Coles, Phys. Rep. 262, 1 (1995).

[7] B. J. Jones, V. J. Martínez, E. Saar, and V. Trimble, Rev. Mod. Phys. 76, 1211 (2004).

[8] T. Tatekawa, astro-ph/0412025.

[9] T. Padmanabhan, gr-qc/0503107.

[10] D. Munshi, V. Sahni, and A. A. Starobinsky, Astrophys. J. 436, 517 (1994)

[11] V. Sahni and S. F. Shandarin, Mon. Not. R. Astron. Soc. 282, 641 (1996)

[12] A. Yoshisato, T. Matsubara, and M. Morikawa, Astrophys. J. 498, 48 (1998).

[13] J. D. Barrow and P. Saich, Classical Quantum Gravity 10, 79 (1993).

[14] F. R. Bouchet, R. Juszkiewicz, S. Colombi, and R. Pellat, Astrophys. J. 394, L5 (1992).

[15] F. R. Bouchet, S. Colombi, E. Hivon, and R. Juszkiewicz, Astron. Astrophys. 296, 575 (1995).
[16] T. Buchert, Mon. Not. R. Astron. Soc. 254, 729 (1992).

[17] T. Buchert and J. Ehlers, Mon. Not. R. Astron. Soc. 264, 375 (1993).

[18] T. Buchert, Mon. Not. R. Astron. Soc. 267, 811 (1994).

[19] P. Catelan, Mon. Not. R. Astron. Soc. 276, 115 (1995).

[20] M. Sasaki and M. Kasai, Prog. Theor. Phys. 99, 585 (1998).

[21] T. Buchert and A. Domínguez, Astron. Astrophys. 335, 395 (1998).

[22] T. Buchert and A. Domínguez, astro-ph/0502318.

[23] T. Tatekawa, Phys. Rev. D 71, 044024 (2005).

[24] S. Adler and T. Buchert, Astron. Astrophys. 343, 317 (1999).

[25] M. Morita and T. Tatekawa, Mon. Not. R. Astron. Soc. 328, 815 (2001).

[26] T. Tatekawa, M. Suda, K. Maeda, M. Morita, and H. Anzai, Phys. Rev. D 66, 064014 (2002).

[27] S. Weinberg, Gravitation and Cosmology (John Wiley \& Sons, Chichester, 1972).

[28] T. Tatekawa, Phys. Rev. D 70, 064010 (2004).

[29] W. H. Press, S. A. Teukolsky, W. T. Vetterling, and B.P. Flannery, Numerical Recipes in Fortran 77: The Art of Scientific Computing (Cambridge University Press, Cambridge, 1992), Sec. 7.6.

[30] J. P. Ostriker and P. Steinhardt, Science 300, 1909 (2003). 